\title{
Convergence des galeries dans les formations salifères Mesures in situ et interprétation
}

Y. KAZAN

Université Libanaise,

Faculté de GénieII,

Département de Génie Civil,

Roumieh Liban

Anciennement G.3S

\section{GHOREYCHI}

G.3S Groupement pour l'étude des Structures

Souterraines de Stockage URA 317 (LMS),

École polytechnique, 91128 Palaiseau Cedex
Cet article est une contribution à létude des structures souterraines dans les milieux viscoplastiques, notamment le sel gemme. Les principaux résultats obtenus pendant quatre années, dans une galerie d'expérimentation aux Mines de potasse d'Alsace, sont présentés. Ces mesures portent sur la température, la convergence des parements et le déplacement du massif. Une corrélation nette entre la variation périodique de la température de l'air et la convergence de la galerie est mise en évidence. Les mesures du déplacement du massif pendant le creusement de la galerie sont commentées. La prépondérance du déplacement différé lors de la première année par rapport aux années suivantes est interprétée par le comportement thermomécanique du massif salifère.

Un modèle analytique simple à géométrie cylindrique est employé en vue d'interpréter les résultats. La loi de

Norton-Hoff comme loi de comportement est validée. Le caractère adimensionnel du modèle permet de proposer une méthodologie pour déterminer les caractéristiques rhéologiques du matériau à partir des résultats in situ. Malgré sa simplicité, ce modèle traduit parfaitement les principaux phénomènes caractéristiques observés lors de I'essai.

\section{Closure of galleries in salt formations In situ measurements and interpretation}

This paper is a contribution to the study of underground structures in viscoplastic media, specially salt rock. Basic results obtained during four years in an experimental gallery belonging to the $\alpha$ Mines de Potasse d'Alsace $n$ are presented. In situ measurements concern temperature, gallery closure, and rock mass displacement. A clear correlation between periodic change of air temperature and gallery closure is shown. Measurements of rock mass displacement during gallery excavation are commented. The fact that time dependent displacement obtained during the first year is more important than that of the following years is explained by thermomechanical behaviour of rock salt.

A simple analytical model of cylindrical geometry is employed to interpret the results. Norton-Hoff constitutive law is then validated. Dimensionless feature of the model allows to suggest a methodology for determination of rheological proprieties of the material using in situ measurements. Despite its simplicity, the model traduces perfectly the basic characteristic phenomena observed during the experiment. 


\section{Introduction}

Historiquement, les premières études sur les structures souterraines dans le sel ont été réalisées à l'occasion de l'exploitation des mines de sel et de potasse. Le comportement viscoplastique du sel a commencé d'être pris en compte à partir des années 70 sur les cavités de stockage d'hydrocarbures. Cependant, l'étude des structures souterraines dans le sel a connu un développement très important durant la dernière décennie grâce aux nombreux essais in situ réalisés en couches ou en dômes de sel aux Etats-Unis (Projet Waste Isolation Pilot Plant: WIPP à New Mexico), en RFA (mine de Asse et site de stockage de Gorleben). Ces essais ont été réalisés en vue d'étudier le stockage de déchets radioactifs. En France, le premier essai in situ dans le sel a débuté en 1984, aux Mines de Potasse d'Alsace (Ghoreychi, 1991).

L'essai thermomécanique CPPS, Champ Proche de Puits de Stockage, est la deuxième expérience in situ réalisée en France dans une formation salifère. Cet essai a été effectué quelques années après l'expérience Dry Dutch Hole réalisée par nos collègues néerlandais dans la mine de sel d'Asse et pratiquement en même temps que l'expérimentation HAW (Highly Active Waste), récemment achevée, également dans la mine de Asse en RFA (GSF, 1995). L'essai, comme l'indique son nom, est destiné à l'étude du champ proche d'un puits de stockage. Il s'inscrit donc dans le cadre des études générales sur le stockage de déchets radioactifs en milieu géologiques entreprises en France préalablement au choix de laboratoires souterrains. Cependant, cet essai comporte de nombreuses mesures expérimentales visant à étudier la tenue mécanique des ouvrages souterrains (galeries et puits) et le comportement du massif encaissant ces ouvrages.

Nous relatons dans cet article les mesures effectuées exclusivement dans la galerie d'expérimentation. Les résultats seront interprétés d'un point de vue géotechnique et thermomécanique. Nous nous efforçons à mettre en relief les principaux phénomènes rencontrés lors de l'essai in situ et à les interpréter par un modèle volontairement simplifié.

\section{2}

\section{Présentation de l'essai in situ}

Après une présentation sommaire du site, de l'instrumentation et du déroulement chronologique de l'essai. nous passerons en revue les mesures effectuées, et commenterons les résultats obtenus afin de dégager les principaux phénomènes rencontrés lors de l'expérimentation.

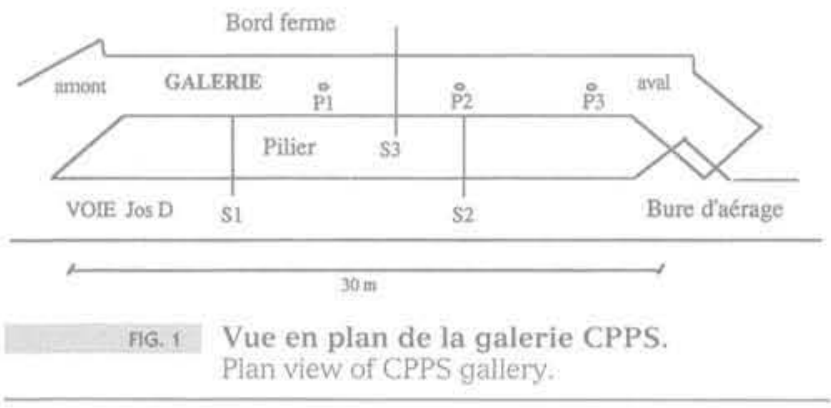

\section{Site de l'essai}

L'essai a eu lieu aux Mines de Potasse d'Alsace (MDPA), où plusieurs mines de sel sont exploitées entre $500 \mathrm{~m}$ et $900 \mathrm{~m}$ de profondeur. Chaque mine est accessible par plusieurs puits de production et d'aérage. L'essai CPPS s'est déroulé dans la mine d'Amélie à $520 \mathrm{~m}$ de profondeur, dans une couche de sel gemme de $20 \mathrm{~m}$ d'épaisseur, où des bancs marno-anhydritiques de faibles épaisseurs s'intercalent avec le sel gemme très pur (plus de $95 \%$ de $\mathrm{NaCl}$ ). En raison de la stratification à faible pendage et de la présence de nombreux intercalaires mamo-anhydritiques, toutes les galeries ont été creusées en sections rectangulaires. L'aire de la section est de $15 \mathrm{~m}^{2}$ en moyenne.

La galerie CPPS a été creusée en remontant depuis une voie préexistante (Jos D), avec une section rectangulaire d'une hauteur de $3 \mathrm{~m}$ et d'une largeur de $4 \mathrm{~m}$ (Fig. 1). Le toit et le mur suivent le pendage des strates de $7 \%$. La partie parallèle à la voie préexistante est de $30 \mathrm{~m}$ de longueur, tandis que la longueur totale de la galerie est de $40 \mathrm{~m}$. La distance entre les axes de la galerie et de la voie Jos D est de $8 \mathrm{~m}$, la largeur du massif qui les sépare, le «Pilier» est de $4 \mathrm{~m}$. En adoptant la terminologie des mineurs, les quatre parties du massif avoisinant seront appelées: le mur, le pilier, le toit et le bord ferme. Les côtés latéraux de la galerie seront appelés: «les parements ».

Le toit a été boulonné pour des raisons de sécurité du fait de la présence des intercalations marno-anhydritiques sub-horizontales. Le soufflage du mur, phénomène observé pendant les six premiers mois qui ont suivi le creusement, a montré l'utilité de cette disposition. Précisons que le soufflage du mur a entraîné un décollement d'un banc de sel gemme de 0,5 m d'épaisseur, conduisant à une hauteur de galerie de $3,5 \mathrm{~m}$. Le creusement de la galerie expérimentale s'est terminé par la réalisation d'un bure d'aérage, s'ouvrant sur la voie Jos D. Ce bure a permis l'aérage de la galerie en la reliant au réseau d'aérage de la mine d'Amélie.

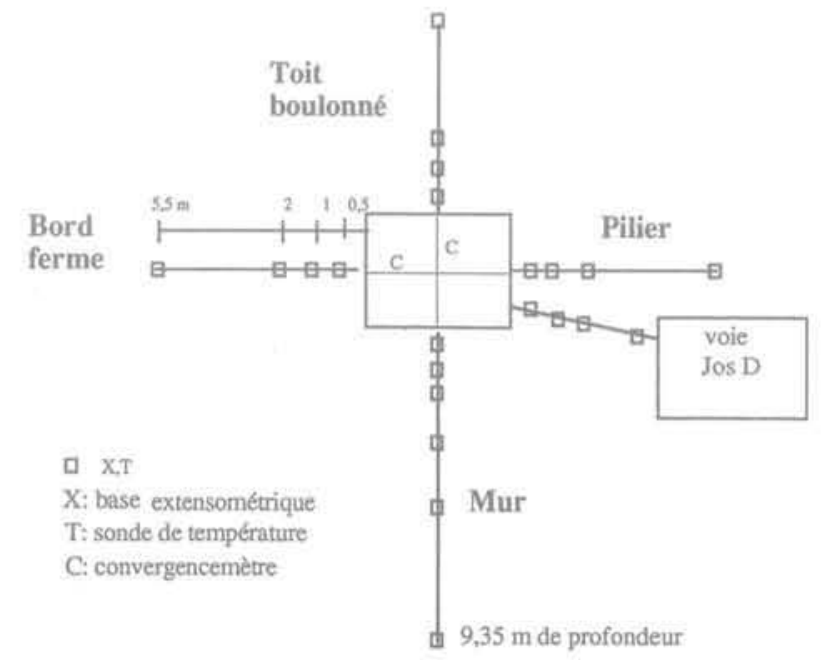

FG. 2 Schéma de certaines bases de mesures de l'essai CPPS.

Scheme of a few bases of measurements of CPPS gallery. 
Distance du front $(\mathrm{m})$

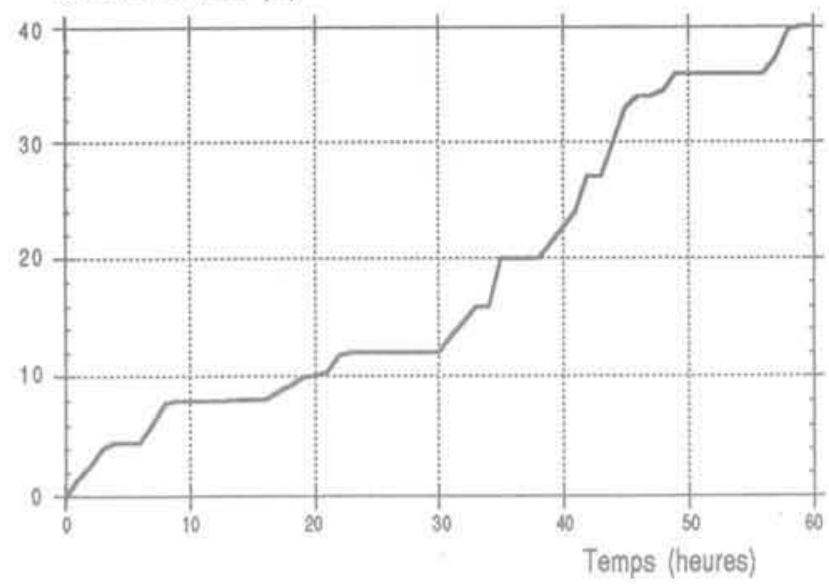

FG.3 Avancement du front de taille de la galerie CPPS,

Advance of CPPS gallery face.

\section{Instrumentation}

L'un des principaux objectifs de l'essai était l'étude de la tenue de la galerie expérimentale et du comportement du massif encaissant. Dans ce but, des capteurs de différentes natures (sondes de température, convergencemètres, bases extensométriques) ont été installés à de nombreux endroits (Fig. 2). Les instruments de mesure ont été spécialement conçus pour être particulièrement précis et peu sensibles à la variation de la température.

Afin de disposer d'instruments de mesure fiables et précis (ne présentant pas de dérive sous température ni dans le temps, et être suffisamment résistants à la corrosion due au contact avec le sel), nous avons été amenés au choix et à la conception des instruments suivants :

- sondes platine à quatre fils (au lieu de thermocouples classiques) permettant de ne pas faire intervenir la longueur des câbles électriques dans la mesure, précision $=0,1^{\circ} \mathrm{C}$. Les sondes ont été couvertes d'une gaine protectrice de la corrosion dans le massif. Le nombre important des sondes platine utilisées montre l'importance accordée à la mesure de température. Ceci est dû au fait que le comportement du sel gemme est très thermo-sensible;

- capteurs de déplacement (précision d'un micromètre) fondés sur le principe de la mesure du champ inductif à partir du déplacement d'un noyau amagnétique au sein de deux bobines. Les tiges d'extensométrie sont en silice ayant un coefficient de dilatation très faible $\left(10^{-7}{ }^{\circ} \mathrm{C}^{-1}\right)$. Ce choix a permis d'assurer que l'élongation thermique ne dépasse pas $1 \mu \mathrm{m} /{ }^{\circ} \mathrm{C}$ pour une longueur de tige de $10 \mathrm{~m}$;

- utilisation de fils «Invar» pour les convergencemètres placés dans la galerie (coefficient de dilatation thermique $=10^{-8}{ }^{\circ} \mathrm{C}^{-1}$ pour les températures inférieures à $40^{\circ} \mathrm{C}$;

- utilisation du système de pilotage et d'acquísition de données SELTICA, conçu spécialement pour les essais in situ par B. Bazargan (Bazargan, 1988).

Les capteurs et l'ensemble du système de mesures ont fait l'objet de nombreuses vérifications avant leur

mise en place in situ. Le choix de l'emplacement des capteurs a été guidé par les objectifs suivants:

- accéder, radialement, à des variations de la température et du déplacement, et vérifier la dissymétrie éventuelle de ces variations au toit, au mur, dans le pilier et au bord ferme de la galerie ainsi que dans l'axe de la galerie et autour des puits destinés aux essais thermomécaniques;

- disposer de bases de mesure les plus proches possibles des parois de la galerie. Dans la pratique, il a fallu s'écarter de quelques décimètres de la paroi, du fait de la déviation inévitable des axes de la galerie et des puits par rapport aux directions définies dans le projet; - disposer de bases de mesure suffisamment éloignées des parois (points « fixes $)$ ). Un compromis consistait à s'éloigner de $5,5 \mathrm{~m}$ dans le toit, le pilier et le bord ferme, et de $9,5 \mathrm{~m}$ au mur, à l'interface entre les bancs de sel gemme et les bancs marno-anhydritiques.

\section{3}

\section{Chronologie de l'essai}

Les résultats présentés ci-après portent sur quatre années successives ( 1461 jours, du 3 avril 1989 jusqu'au 2 avril 1993). L'instrumentation du site expérimental a débuté par l'installation de deux tiges extensométriques obliques, équipées chacune de quatre bases extensométriques dans les sections S1 et S2, mises en place depuis la voie Jos D. Le but de ces capteurs est de mesurer le déplacement élastique instantané du massif dû au creusement, ainsi que le déplacement dû au fluage primaire du massif. Faute de courant disponible lors du creusement, du fait de l'impératif de sécurité de la mine (grisou), les mesures ont été effectuées manuellement pendant le creusement.

Le creusement de la galerie, assuré par une machine à attaque ponctuelle, a duré soixante heures (du 3 avril 1989 à 8 heures jusqu'au 5 avril à 19 heures), pour une longueur totale de $40 \mathrm{~m}$. La figure 3 montre l'avancement du creusement en fonction du temps.

Une fois le creusement achevé, le toit a été boulonné avant qu'on installe les capteurs de mesure. La phase préliminaire du suivi du comportement du massif, a commencé quelques jours après pour durer six mois environ, pendant lesquels le phénomène de soufflage du mur a été observé. Alors, les travaux dits de rabassenage (dans le jargon minier) du mur ont ramené la hauteur de la galerie à $3,5 \mathrm{~m}$. Trois puits verticaux ont été ensuite forés au mur de la galerie pour y installer des sondes chauffantes. Le premier essai de chauffe a commencé au jour 1100 . Son effet est manifeste sur la plupart des courbes de mesure.

\section{3}

\section{Principaux résultats}

\section{1}

\section{Creusement de la galerie}

L'extensométrie a été réalisée, comme nous l'avons déjà précisé, même lors de l'excavation de la galerie au moyen de deux extensomètres obliques, implantés à partir de la voie d'accès Jos D, dans ce qui est devenu 
Déplacement $(\mathrm{mm})$

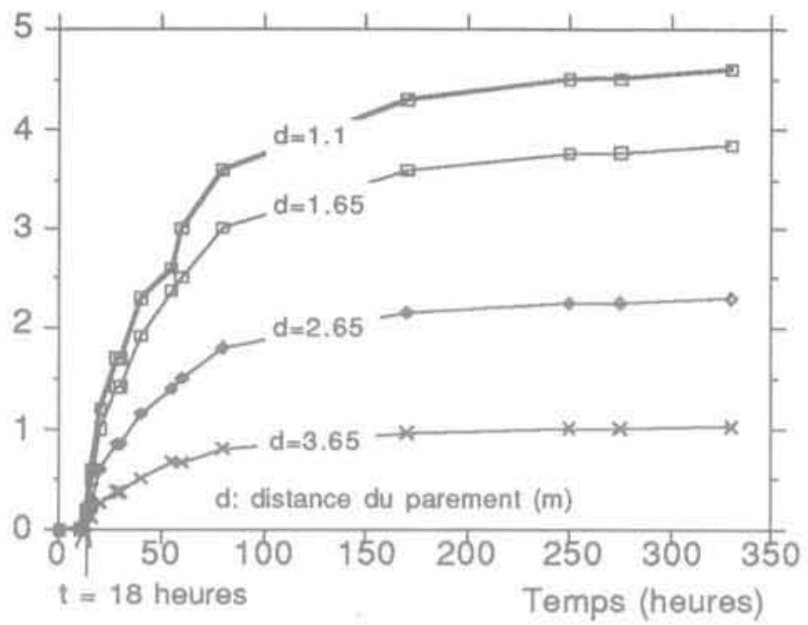

a - Section S1
Déplacement (mm)

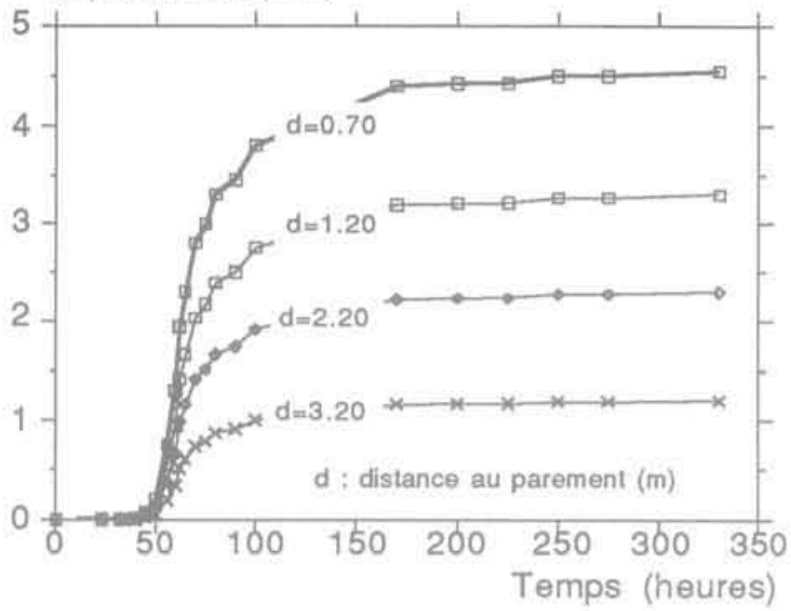

b - Section S2

FiG.4 Déplacements du massif depuis le creusement de la galerie CPPS. Rock mass displacement since the excavation of CPPS gallery.

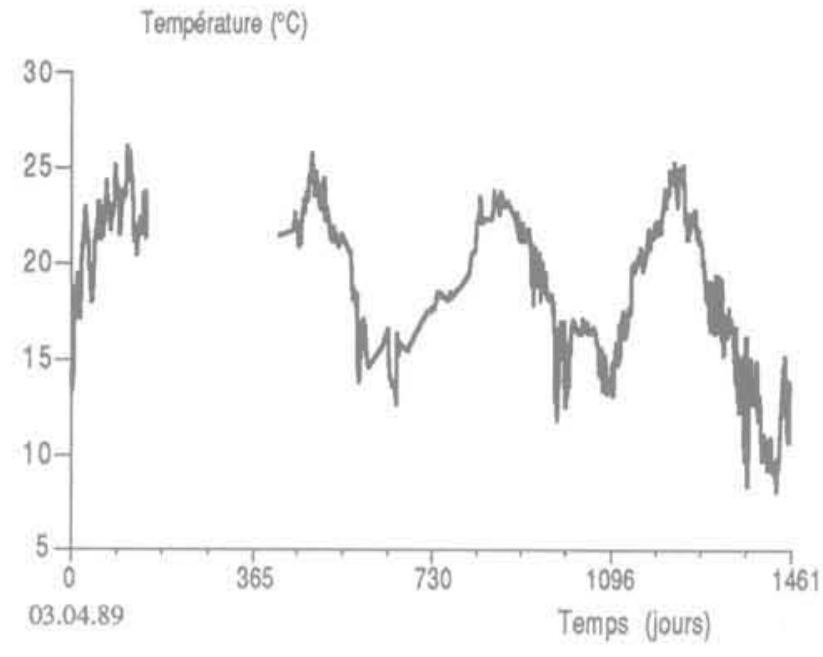

FG. 5 Température ambiante de l'air de la galerie CPPS.

Air temperature of CPPS gallery. après l'excavation le pilier. Les figures $4 \mathrm{a}$ et $4 \mathrm{~b}$ montrent respectivement le déplacement du massif dans les sections S1 et S2, repérées sur la figure 1, pendant les deux premières semaines qui ont suivi le creusement de la galerie CPPS.

Nous remarquons que le déplacement du massif dans la section S1, située à 9,5 m à l'entrée de la galerie, commence à devenir perceptible au bout d'environ 18 heures, bien avant celui de $\mathrm{S} 2$, située à $22,5 \mathrm{~m}$ de l'entrée de la galerie. Le déplacement de cette dernière section commence à être significatif au bout de 40 heures. Ces deux instants correspondent à l'arrivée du front de taille à ces sections (voir Fig. 4). Le déplacement d'une section n'est perceptible qu'après l'arrivée du front de taille à cette section. Ainsi, ces mesures correspondent au déplacement élastique et au fluage primaire du massif qui se stabilise au bout d'une semaine environ. Les déplacements différés dus au fluage

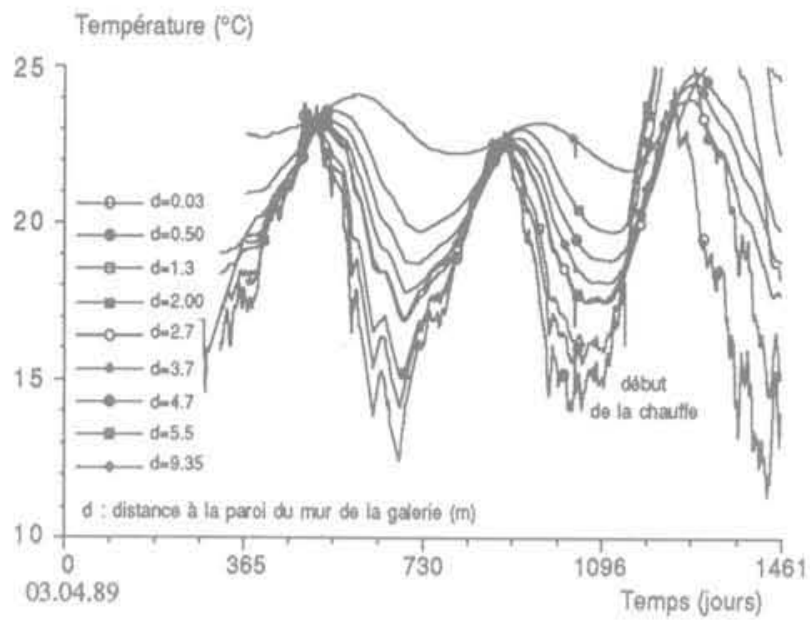

FG.6 Température au mur de la galerie CPPS. Roof temperature of CPPS gallery. secondaire n'ont pas encore pu être détectés par les capteurs.

\section{2}

\section{Température}

Rappelons que la température naturelle du massif à la profondeur de la galerie est de $36^{\circ} \mathrm{C}$. La figure 5 présente l'évolution de la température ambiante de l'air de la galerie CPPS. On y reconnaît une allure périodique à l'échelle de plusieurs années: oscillation autour de $16^{\circ} \mathrm{C}$ avec un minimum de $7^{\circ} \mathrm{C}$ en hiver 1993 et un maximum de $26^{\circ} \mathrm{C}$ pendant les étés.

Au sein d'une période d'un an, on observe des variations plus ou moins irrégulières mensuelles, hebdomadaires voire même journalières. Ces variations se 
Température $\left({ }^{\circ} \mathrm{C}\right)$

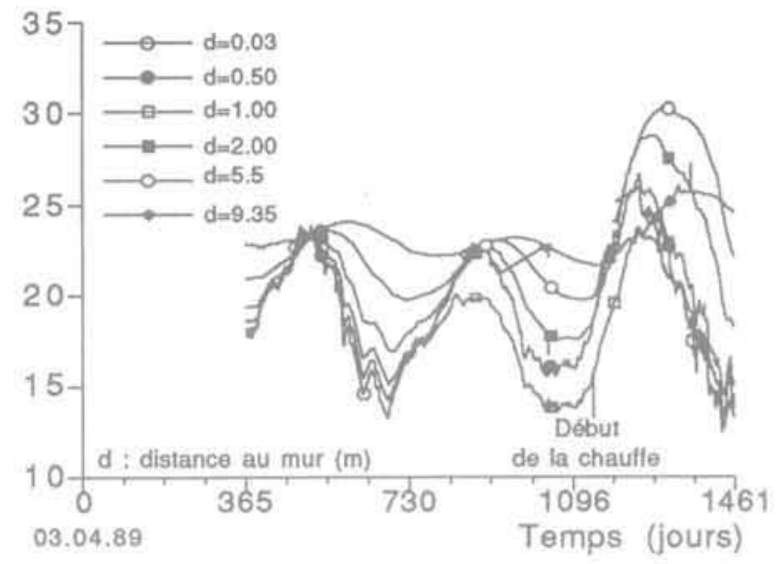

a - mur

Température $\left({ }^{\circ} \mathrm{C}\right)$

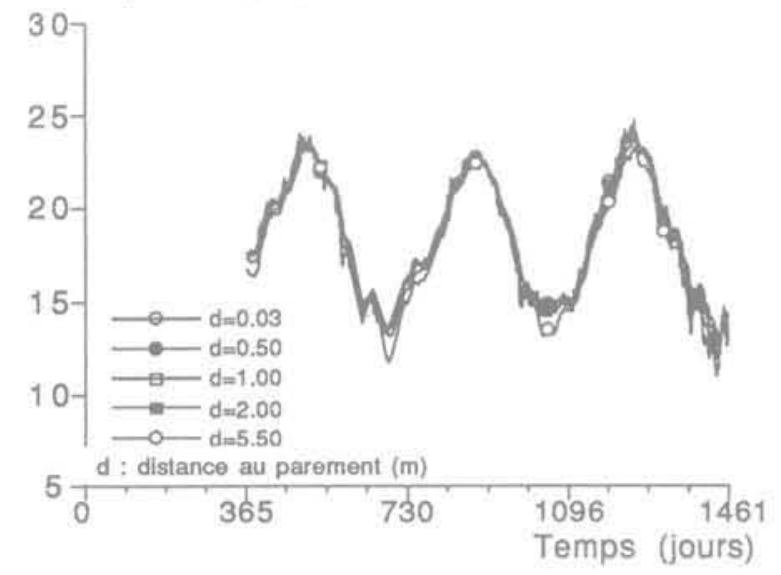

c- pilier
Température $\left({ }^{\circ} \mathrm{C}\right)$



b - toit

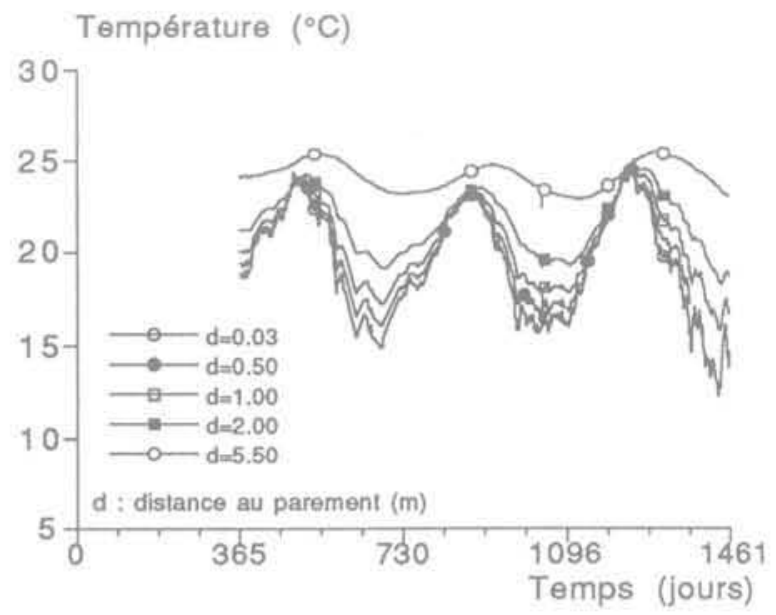

d - bord ferme

FIG.7 Température dans les quatre parties du massif. Temperature in four parts of rock mass.

manifestent même aux points les plus éloignés de la paroi de la galerie, à une dizaine de mètres à l'intérieur du massif (Fig. 6). Bien que les variations s'atténuent au fur et à mesure que lion s'éloigne de la paroi, la périodicité de la température ambiante est encore bien visible à $9,35 \mathrm{~m}$ de profondeur par des variations de faible amplitude. Ce phénomène tient à la grande diffusivité thermique du sel gemme $\left(3.10^{-6} \mathrm{~m}^{2} / \mathrm{s}\right.$ soit trois fois plus importante que la moyenne des géomatériaux).

Une comparaison entre la température de l'air et celle de la paroi de la galerie montre que la continuité de la température n'est pas assurée entre l'air et la paroí. de la galerie. Ce phénomène, caractéristique d'un échange thermique par convection entre l'air et le massif, tient à l'aérage de la galerie. Il est à noter que la vitesse de l'air n'est pas très élevée dans la galerie CPPS. Une vitesse de ventilation d'air beaucoup plus élevée (théoriquement infinie) conduirait à la continuité de la température sur la paroi, tandis qu'une vitesse nulle aboutirait à des écarts plus importants.
Concernant les différentes parties du massif, on note, comme il est normal, que le pilier se distingue des autres parties du fait de son exposition simultanée aux aérages des deux galeries quil le délimitent (Fig. 7). Néanmoins, à quelques degrés près, le champ de température est identique aux quatre côtés du massif, à condition toutefois d'exclure la période postérieure au chauffage du puits (jour 1100) qui affecte considérablement le comportement du mur de la galerie.

\section{3}

\section{Convergence de la galerie}

Du fait de sa facilité et de sa fiabilité, la mesure de convergence est largement utilisée en géotechnique. Cette mesure revêt un intérêt particulier dans le cas du sel gemme, matériau qui ne possède en principe aucun seuil de viscoplasticité. II s'ensuit que toute cavité non 


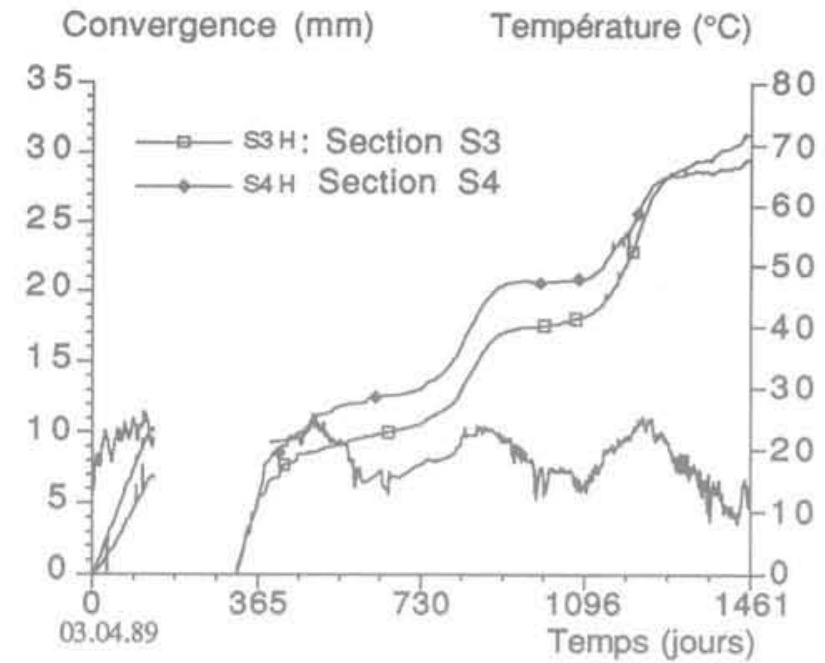

HG. e Convergence horizontale de la galerie CPPS.

Horizontal closure of CPPS gallery. soutenue creusée en milieu salifère se déforme jusqu'à sa fermeture totale. La vitesse de convergence constitue une donnée essentielle caractérisant la stabilité à long terme de l'ouvrage. Une vitesse modérée (typiquement inférieure à $1 \%$ par an pour fixer les idées) conduit rarement à des désordres mécaniques (écaillage de la paroi, effondrement de l'ouvrage), alors qu'une vitesse élevée (plusieurs pour cent par an) aboutit souvent à une instabilité mécanique de l'ouvrage à long terme.

Sur les figures 8 et 9 sont rapportées les convergences, horizontales et verticales, mesurées durant quatre ans dans deux sections, S3 et S4. Nous remarquons, dans toutes les bases de mesure, une certaine stabilité de la vitesse de convergence. Au cours des deux dernières années du suivi, la convergence est
Convergence $(\mathrm{mm})$

Temperature $\left({ }^{\circ} \mathrm{C}\right)$

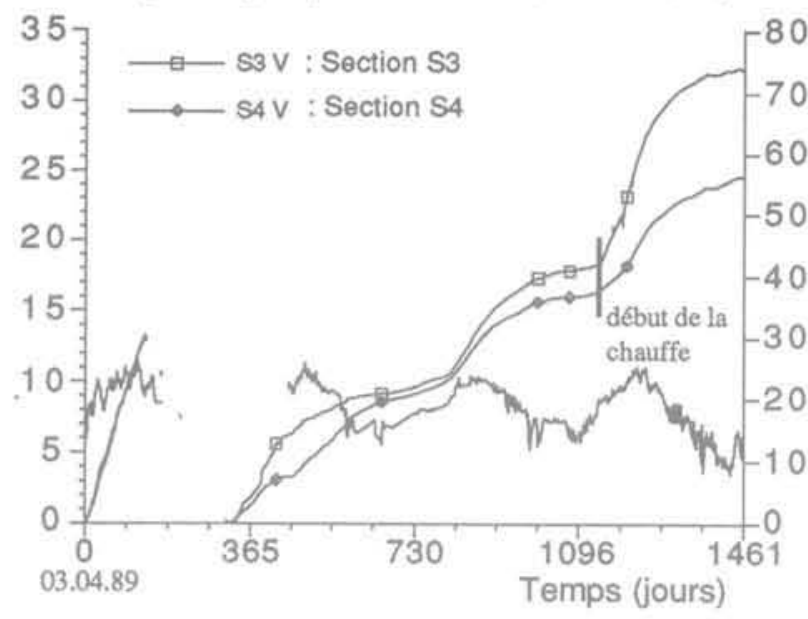

FIG. 9 Convergence verticale de la galerie CPPS. Vertical closure of CPPS gallery.

d'environ $10 \mathrm{~mm} / \mathrm{an}$, soit $0,25 \%$ par an en moyenne (section de la galerie $3,5 \mathrm{~m} \times 4 \mathrm{~m}$ ). C'est une vitesse de convergence relativement faible qui témoigne de la bonne tenue mécanique de la galerie CPPS.

On remarque également un écart peu important entre les déplacements horizontaux et verticaux. Cet écart est lié à l'anisotropie du champ de contraintes initiales régnant autour de la galerie (la galerie est creusée dans une mine et non au sein d'un massif infini), à l'anisotropie des terrains sédimentaires ainsi qu'à l'absence d'homogénéité du champ de température autour de la galerie. Il convient de noter une accélération de la convergence verticale après le démarrage de la chauffe. Ce phénomène est lié au soulèvement du mur de la galerie par suite de la dilatation thermique du sel.
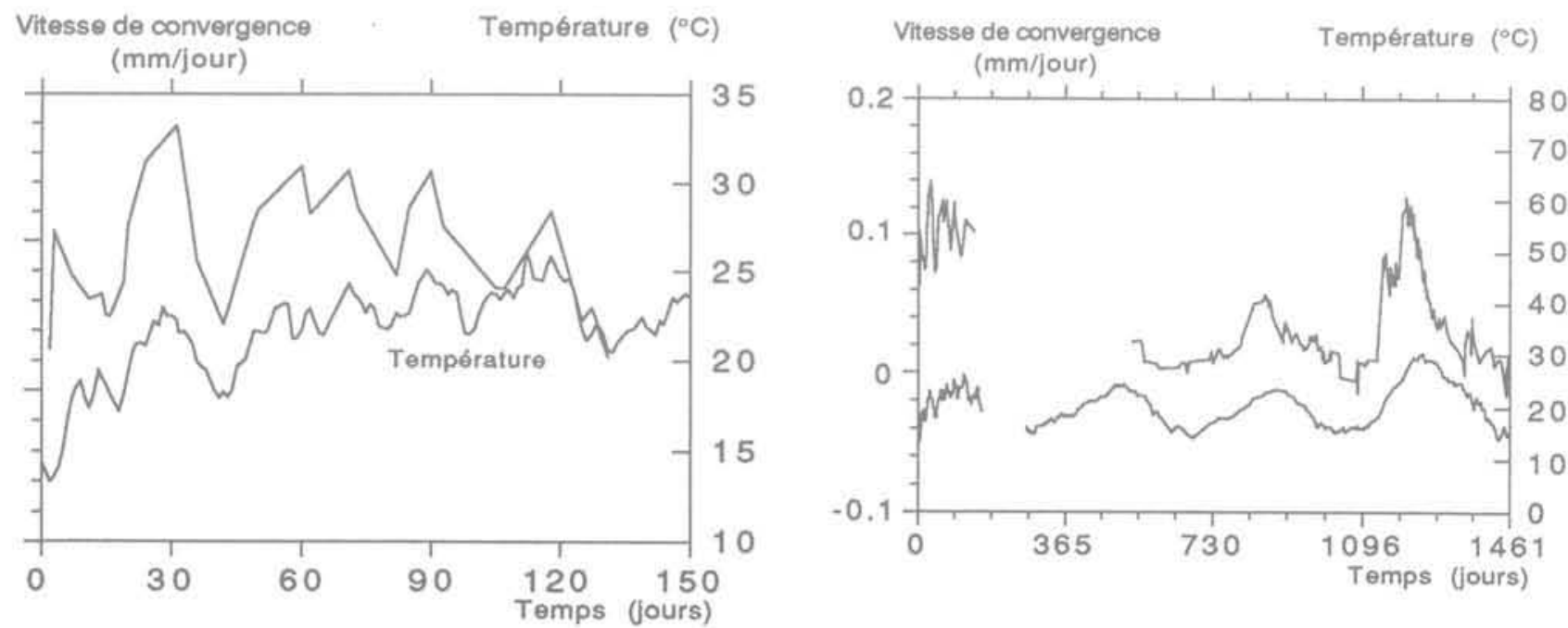


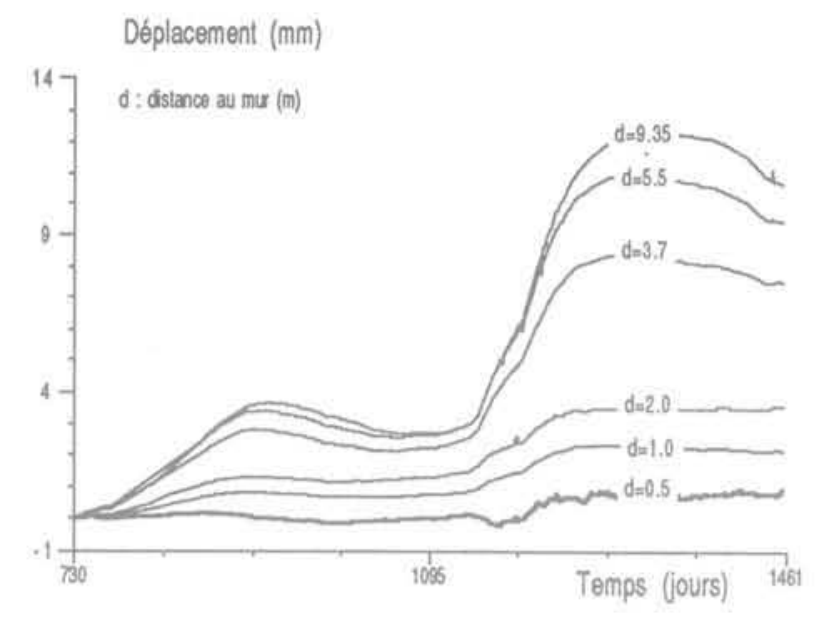

FIG. 11 Déplacement relatif du mur par rapport à la paroi de la galerie CPPS.

Relative displacement of the floor with respect to the wall of CPPS gallery.
Mais le phénomène le plus significatif est la corrélation entre la vitesse de la convergence et la température ambiante (voir Fig. 10) : la convergence s'accélère en été et s'estompe pratiquement en hiver. Mais, aucune divergence de la paroi n'est cependant observée. Ce phénomène montre combien le comportement mécanique du sel d'Amélie est sensible à la température. Notons que la vitesse de convergence pendant l'été de la première année est d'environ $0,1 \mathrm{~mm} / \mathrm{jour}$, tandis qu'elle ne dépasse pas $0,04 \mathrm{~mm} / \mathrm{jour}$ pendant la troisième année. L'augmentation brusque de la vitesse de convergence durant la quatrième année de l'essai est due à l'essai de chauffage réalisé dans un forage au mur.

La mesure de convergence fournit le déplacement relatif de deux points sur les parements opposés, mais ne permet pas, à elle seule, d'identifier l'origine du déplacement prépondérante. C'est pourquoi ces mesures ont été complétées par des mesures d'extensométrie au sein du massif.

\section{4}

\section{Extension du massif}

L'extension du massif a été mesurée, après le creusement, dans les quatre parties du massif encaissant la section médiane S3 à différentes distances de la paroi. Pour des raisons techniques, les points d'ancrage des tiges extensométriques ont été placés à la paroi de la galerie. Ainsi, chaque tige suit entièrement le déplacement $\mathrm{U}_{0}$ de la paroi. De ce fait, les mesures brutes fournies par les bases extensométriques $v_{\text {, correspondent à }}$ des déplacements relatifs par rapport à la paroi. La détermination du déplacement de la paroi serait alors indispensable pour connaître le déplacement réel du massif. Une approximation simple consiste à négliger le déplacement de la base la plus éloignée du mur de la galerie. Cette hypothèse est d'autant plus vraisemblable que la dernière base se situe assez loin de la paroi de la galerie.
Les courbes de la figure 11 correspondent au déplacement de six bases d'une tige extensométrique située au mur de la galerie. Ces déplacements étant comptés à partir de la paroi, nous comprenons pourquoi les valeurs sont d'autant plus grandes que la base considérée se trouve loin. Les courbes correspondant aux distances de $9,35 \mathrm{~m}$ et $5,5 \mathrm{~m}$ coïncident presque parfaitement (hors la période de chauffe). Ce fait montre que les déplacements de ces bases sont négligeables par rapport à celui de la paroi. Ainsi, nous pouvons confondre les déplacements de la paroi avec les valeurs signalées par les bases les plus éloignées. Pour s'assurer de la validité de cette supposition, il reste à vérifier que la somme des déplacements des deux parois opposées correspond bien aux valeurs mesurées par les convergencemètres.

Les figures $12 \mathrm{a}$ et $12 \mathrm{~d}$ correspondent aux mesures réalisées pendant les cinq premiers mois qui ont suivi le creusement de la galerie. On s'aperçoit que le déplacement immédiat du mur est prépondérant par rapport aux déplacements des autres parois, ce qui s'explique par le phénomène de soufflage du mur survenu sur le site de l'essai. Les déplacements des bases situées dans le pilier sont bien plus grands que ceux du toit et du bord ferme: ce qui est probablement dû à la concentration de contraintes déviatoriques dans le pilier.

Les figures 13a à 13d présentent les mesures de déplacements réalisées pendant les deux dernières années de l'essai. Cette période commence après la remise en état du mur de la galerie rendu nécessaire par le décollement du premier banc. On remarque des déplacements centimétriques cumulés pendant les deux dernières années. Ces valeurs sont compatibles avec la convergence et confirment la fiabilité de l'ensemble des mesures de déplacements.

\section{4}

\section{Interprétation des mesures}

En nous limitant exclusivement à l'analyse du champ de déplacement, nous allons interpréter les résultats de l'essai in situ par un modèle analytique simplifié, celui d'une cavité cylindrique soumise à la température, dans un milieu viscoplastique de NortonHoff.

Tenter d'interpréter les résultats de l'essai CPPS à partir d'un modèle analytique simple est un pari difficile. En effet, le milieu est loin d'être homogène et isotrope, l'isotropie du champ de contraintes initiales n'est pas assurée, la galerie se trouve à proximité des ouvrages voisins, et sa section est rectangulaire. Il est donc évident qu'une modélisation fine ne peut être effectuée que par un calcul numérique aux éléments finis par exemple. Mais, nous choisissons volontairement la voie analytique en visant les deux objectifs suivants :

- démontrer qu'en dépit de leurs complexités, certains aspects du comportement des structures souterrains (phénomènes, ordres de grandeurs...) peuvent être compris et analysés plus facilement par des modèles simples :

- dégager de l'essai in situ, des renseignements généraux utiles à l'analyse des comportements thermomécaniques de tout ouvrage souterrain dans le sel. 


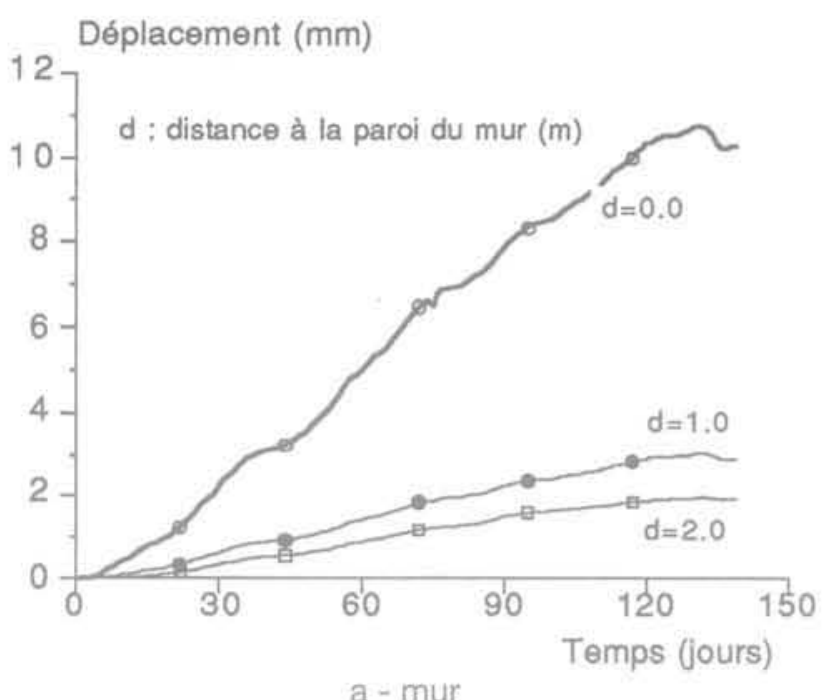

a - mur
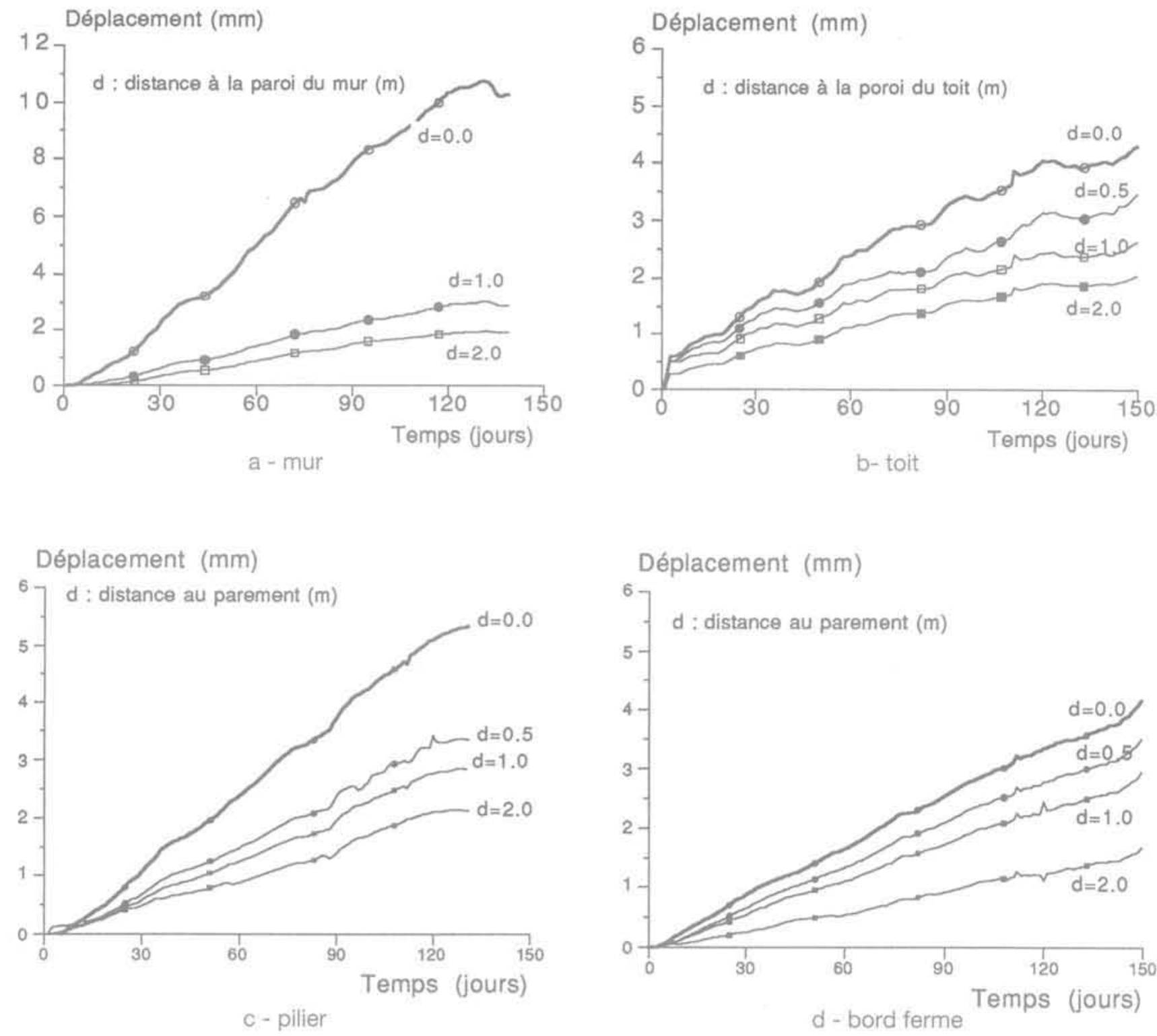

FiG. 12 Déplacement du massif durant les cinq premiers mois de l'essai. Rock mass displacement during the first five months.

\section{1}

\section{Constatations expérimentales}

L'analyse globale des courbes de convergence de la galerie révèle quelques caractéristiques permettant l'utilisation d'un modèle analytique simple (cavité cylindrique), objet du paragraphe 4.3. Les faits suivants sont en particulier constatés:

1. Uniformité relative des deux convergences, verticale et horizontale. Cette observation permet de simuler la galerie, en première approximation, par une cavité cylindrique de rayon égal à $2 \mathrm{~m}$;

2. Stabilité de la convergence moyenne au cours des dernières années de l'essai $(0,25 \% / a n)$. La convergence étant prépondérante pendant la première année. Cette observation permet de justifier l'adoption de la loi de Norton-Hoff, comme loi de comportement du sel étudié. Cette loi est en mesure d'interpréter la légère diminution progressive de la vitesse de convergence rela- tive aux mêmes périodes saisonnières, au cours des différentes années. En effet, le calcul montre une diminution continue de la vitesse de convergence de la structure, même si la loi de comportement utilisée repose sur le concept de fluage stationnaire, valable pour l'essai de fluage réalisê au laboratoire;

3. Amplitude négligeable de la convergence due au fluage primaire. Ce fait, mis en évidence par les extensomètres obliques mesurant le déplacement du massif au creusement de la galerie, a déjà été commenté; 4. Sensibilité considérable à la température. La vitesse de convergence, marquée par l'influence de la variation de la température, en oscillant autour d'une moyenne de $0,05 \mathrm{~mm} /$ jour, s'annule en hiver (Fig, 8 et 9 ).

Ce dernier phénomène n'est pas lié seulement à la variation de la viscosité en fonction de la température. En effet, l'évolution de la viscosité du sel gemme en fonction de la température suit la loi d'Arrhénius d'expression exponentielle décroissante. En prenant une valeur typique de la constante d'activation de 


\section{Déplacement (mm)}

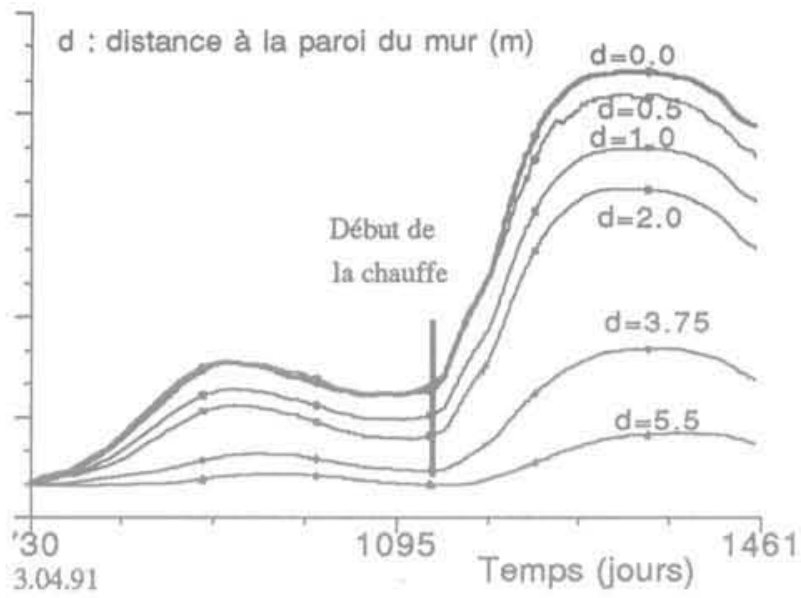

a - mur

\section{Déplacement $(\mathrm{mm})$}

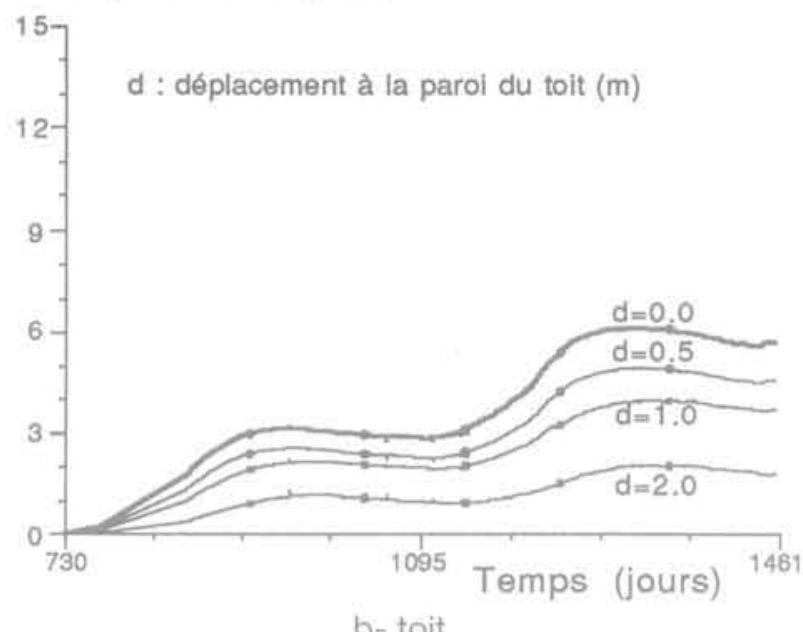

Déplacement $(\mathrm{mm})$

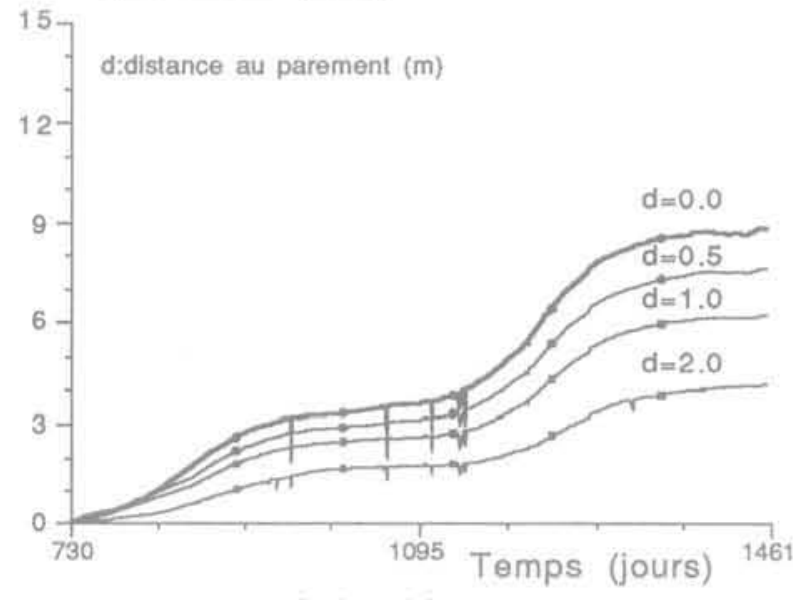

d - bord ferme

\section{Déplacement (mm)}

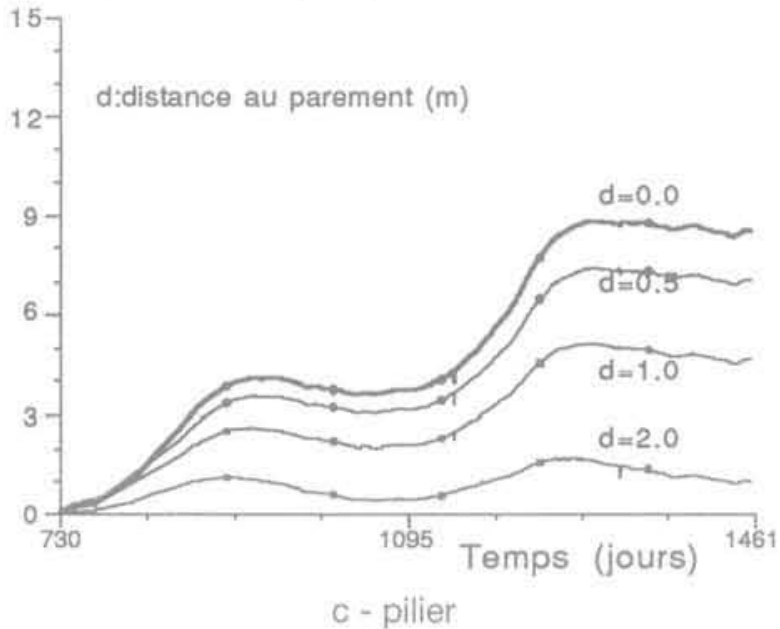

FIG. 13 Déplacement du massif durant les deux dernières années de l'essai. Rock mass displacement during the last two years of the test.

5600 Kelvin pour la loi d'Arrhénius, une variation de température de $\pm 10^{\circ} \mathrm{C}$ conduirait à une modification du coefficient de fluidité dans l'intervalle [0,5 à 2], soit un rapport de 4 alors que la vitesse in situ varie dans un rapport pratiquement infini. Nous verrons dans le paragraphe suivant que cet écart important provient réellement de la variation de la contrainte déviatorique dans le massif. En effet, le déviateur g en paroi de la galerie est composé de deux parties, d'origines mécanique et thermique

$$
g=g_{\text {mec }} \pm \frac{E \alpha}{1-v} T_{0} f(t)
$$

$\mathrm{T}_{0}$ étant l'amplitude de la variation de la température, $\mathrm{f}(\mathrm{t})$ fonction qui tient compte des déformations viscoplastiques. Ainsi, en hiver, des contraintes thermiques négatives en valeur absolue (contraintes de traction liées au refroidissement) viennent diminuer considérablement le déviateur jusqu'à l'annuler. Ce phénomène, qui sera illustré plus loin par un calcul thermomécanique, est à l'origine de la diminution notable de la vitesse de convergence pendant l'hiver.

\section{4,2}

\section{Hypothèses simplificatrices}

\section{4,89}

Milieu

Le site de l'essai se trouve dans un banc de sel gemme traversé par des lits marno-anhydritiques d'épaisseur centimétrique. Le banc de sel s'étend sur quelques mètres au toit et au mur de la galerie. Nous proposons d'assimiler, d'une manière simplifiée, l'ensemble des terrains encaissant la galerie CPPS à un banc équivalent défini de la façon suivante: 


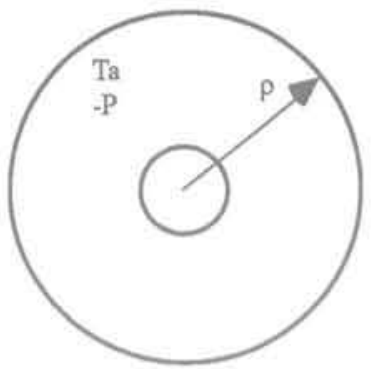

Etat initial

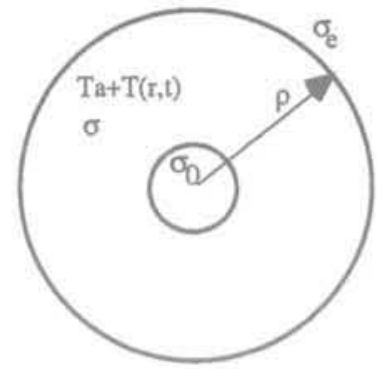

Etat sous chargement
हiG. 14 Schématisation du problème en géométrie cylindrique.

Idealisation of the problem with cylindrical geometry.

a) continu (d'un point de vue de déplacement). C'est une hypothèse valable, confirmée par les mesures d'extensométrie (le seul décollement survenu étant le soufflage du premier banc du mur, juste après le creusement de la galerie);

b) isotrope (d'un point de vue de propriétés mécaniques). Cette hypothèse, discutable dans tout terrain sédimentaire, va en général de pair avec une certaine homogénéisation du massif;

c) milieu salifère infini. Les mesures de déplacement mettent en évidence que le comportement du massif est déterminé par celui des bancs de sel gemme. Les déplacements se poursuivent d'une manière plus ou moins stationnaire, ce qui est dû au fluage du sel gemme. En outre, l'épaisseur du banc de sel est d'environ trois fois la hauteur de la galerie.

\section{2}

\section{Contraintes naturelles}

Étant unanimement admis que le sel est théoriquement dépourvu de tout seuil d'écoulement viscoplastique, on ne peut pas admettre, à l'échelle géologique, l'existence d'un déviateur de contraintes naturelles dans un massif salifère homogène suffisamment étendu. Cette situation peut être le cas d'un dôme de sel, mais non nécessairement d'un gisement de sel en couches stratifiées.

Par ailleurs, les valeurs des convergences mesurées dans la galerie dans les direction verticale et horizontale sont très proches (l'écart n'excède pas $10 \%$ ). C'est pourquoi, nous assimilons l'état de contraintes régnant dans le massif avant le creusement de la galerie CPPS à un champ isotrope. Ce champ correspond à une contrainte moyenne résultant du poids du recouvrement (environ $10 \mathrm{MPa}$ à $500 \mathrm{~m}$ de profondeur).

\section{3}

\section{Modèles analytiques}

\section{3 .3}

\section{Hypothèses et équations}

Nous traitons le problème d'une structure cylindrique de longueur supposée infinie, et de rayons inté- rieur et extérieur respectivement $r_{0}=1$ et $r_{e}=\rho$. L'état initial est caractérisé par un champ de contraintes isotrope $\underline{\underline{\sigma_{i}}}=-1$, et par des déformations initiales nulles $\left(\underline{\underline{\varepsilon_{1}}}=0\right)$. Le champ de température initiale est supposé également uniforme $\left(\mathrm{T}_{\mathrm{i}}=\mathrm{T}_{\mathrm{g}}\right)$. La structure subit un chargement mécanique sous forme de contraintes radiales $\sigma_{0}$ et $\sigma_{e}$ imposées respectivement sur les parois intérieure et extérieure (Fig. 14). Elle subit également un chargement thermique caractérisé par une variation de température $\mathrm{T}=\mathrm{T}(\mathrm{r}, \mathrm{t})$. La symétrie géométrique de la structure et des chargements, mécanique et thermique, permet de représenter l'espace par la seule variable radiale $r$. Le problème est alors axisymétrique et en déformations planes.

Nous adoptons les hypothèses suivantes qui s'expriment par les équations du cadre 1 :

H1: petites perturbations:

H2: partition des déformations: La déformation totale en tout point de la structure est la somme de deux déformations : élastique et anélastique;

H3: élasticité homogène, linéaire et isotrope: La déformation élastique est liée au champ de contraintes donné par la loi de Hooke;

$\mathrm{H} 4$ : isotropie thermique: Le coefficient de dilatation thermique est un scalaire $\alpha$.

Le problème comporte sept inconnues: le déplacement radial $u$, trois composantes de contraintes : $\sigma_{r}, \sigma_{\theta^{\prime}}$ et $\sigma_{z}$, et trois composantes de déformations anélastiques: $\varepsilon_{p}^{n}, \varepsilon_{\theta}^{\text {an }}$, et $\varepsilon_{2}^{m}$. Le système d'équations du cadre 1 , comprenant quatre équations, permet d'exprimer le déplacement et les contraintes en fonction des déformations anélastiques; E, v et $\alpha$ étant le module d'élasticité, le coefficient de Poisson et le coefficient de dilatation thermique. Le choix de la loi de comportement anélastique fournira les équations supplémentaires.

Le problème revient à résoudre un système de quatre équations à quatre inconnues, les trois composantes de déformations anélastiques étant considérées comme paramètres (cadre 1). Les équations - solutions du système sont classées dans le cadre 2. Les démarches de résolution du système d'équations sont détaillées dans (Kazan, 1994).

\section{3 .2}

\section{Loi de comportement}

En dehors de quelques lois, proposées dans le temps, considérant la plasticité du sel (Hardy, 1959, Serata, 1964), deux principales familles de loi, fondées sur deux écoles de pensée parmi les plus répandues à travers le monde, font l'objet de l'étude. La première considère une stabilisation de la vitesse de déformation viscoplastique au-delà d'une phase de fluage primaire (ou transitoire). Cette famille, qui est connue sous le nom de "Norton-Hoff ») (Langer, 1981; Wawersik, 1981), admet donc l'existence du fluage stationnaire (établi). La deuxième famille considère en revanche une diminution progressive de la vitesse de déformation et traduit la rhéologie du sel par des lois appartenant à la famille "Lemaître-Menzel-Schreiner» (Menzel et Schreiner, 1977; Vouille et al., 1983; Lux et Heusermann, 1983; Piper, 1989), caractérisée par l'écrouissage. Toutefois, les deux lois s'accordent sur les traits principaux du comportement thermomécanique du sel gemme et admettent que: 
Système d'équations

System of equations

$\begin{array}{ll}E \frac{\partial u}{\partial r}=\sigma_{r}-v\left(\sigma_{\theta}+\sigma_{2}\right)+E \varepsilon_{r}^{a n}+E \alpha T(r, t)+P(1-2 v) & \text { (a) } \\ E \frac{u}{r}=\sigma_{\theta}-v\left(\sigma_{r}+\sigma_{2}\right)+E \varepsilon_{\theta}^{a n}+E \alpha T(r, t)+P(1-2 v) & \text { (b) } \\ 0=\sigma_{z}-v\left(\sigma_{r}+\sigma_{\theta}\right)+E \varepsilon_{2}^{a n}+E \alpha T(r, t)+P(1-2 v) & \text { (c) } \\ 0=\frac{\partial \sigma_{r}}{\partial r}+\frac{\sigma_{t}-\sigma_{\theta}}{r} & \text { (d) }\end{array}$

Équations - Solutions

Equations - Solutions

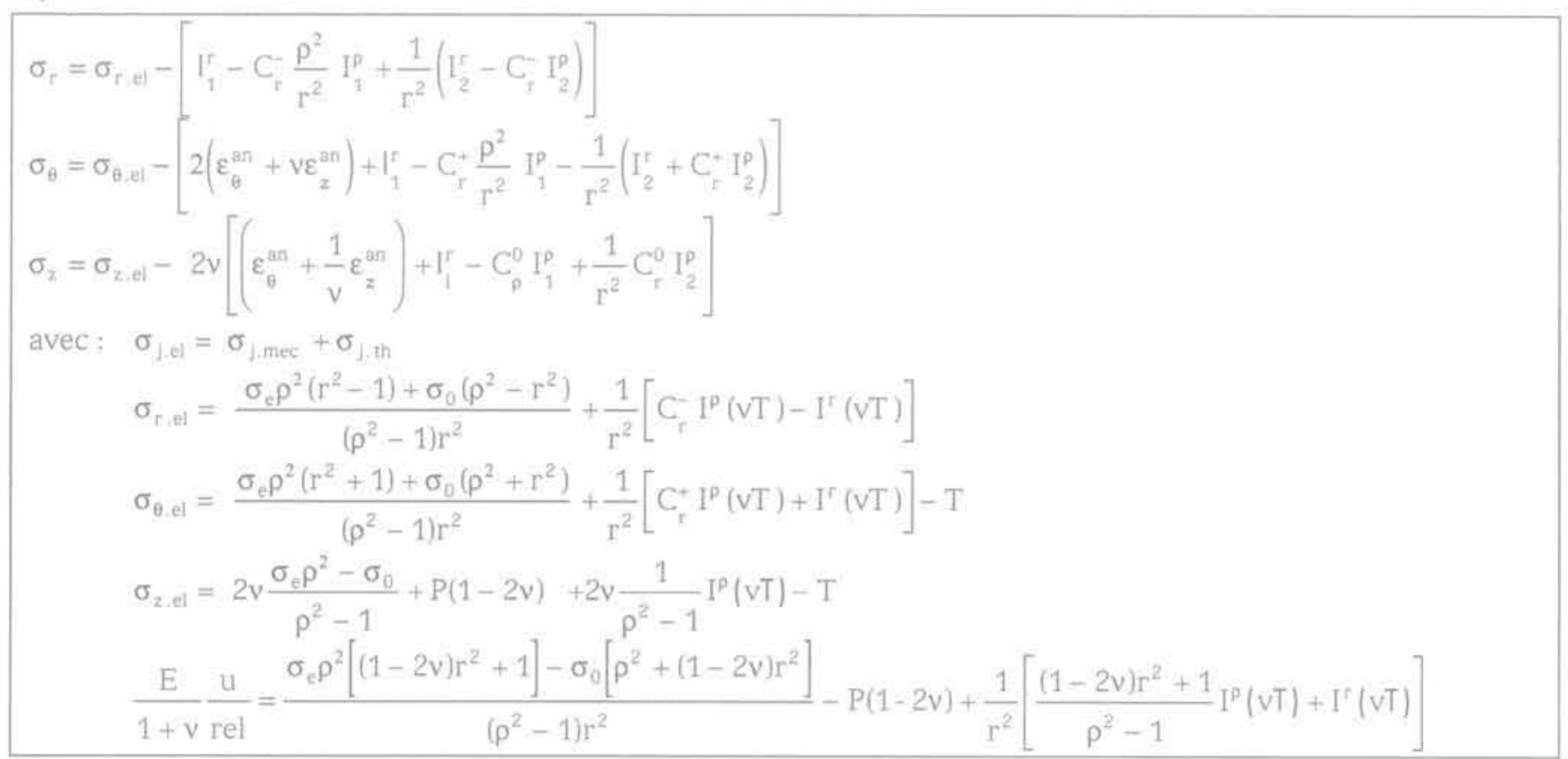

Définition des grandeurs adimensionnelles

$$
\begin{aligned}
& T=\frac{E \alpha}{1-v} T ; \varepsilon_{j}^{a n}=\frac{E}{2\left(1-v^{2}\right)} \varepsilon_{j}^{a n} j=r, \theta \text { ou z } \quad I^{x}(f)=\int_{1}^{x} f(v) d v \\
& C_{r}^{ \pm}=\frac{r^{2} \pm 1}{\rho^{2}-1} ; C_{r}^{0}=\frac{r^{2}}{\rho^{2}-1} ; I_{1}=I\left[v^{-1}\left(\varepsilon_{\theta}^{a n}-\varepsilon_{r}^{a n}\right)\right] ; I_{2}=I\left[v\left(\varepsilon_{r}^{a n}+\varepsilon_{\theta}^{a n}+2 v \varepsilon_{z}^{a n}\right)\right]
\end{aligned}
$$

- la viscosité du sel décroit rapidement avec la température. Elle s'exprime par la loi d'Arrhénius où T est la température absolue et $\mathrm{K}$ la constante d'activation:

$$
\eta=A \exp -\frac{K}{T}
$$

- le seuil de viscoplasticité est nul, ce qui signifie que toute modification non hydrostatique de l'état de contrainte entraîne le fluage. Le comportement différé dépend de la partie déviatorique du tenseur de contraire (et non de la partie sphérique);

- la déformation viscoplastique se produit à volume constant.

Il existe, à l'heure actuelle, un nombre élevé d'expressions mathématiques de loi de comportement pour le sel. La plus simple et sans doute la plus répandue, est celle de Norton-Hoff sans fluage primaire. Les paramètres sont obtenus par une minimisation, au sens des moindres carrés (Nadai, 1938), des données issues de divers essais de fluage. Les lois ainsi exprimées sont généralisées à trois dimensions en adoptant la règle de normalité et en utilisant le critère de viscoplasticité de Tresca:

$$
\dot{\varepsilon}_{i j}^{v p}=\eta(T) \cdot F^{n} \cdot \frac{\partial F}{\partial \sigma_{i j}} \text { avec } F=\left|\sigma_{1}-\sigma_{3}\right|
$$

$\sigma_{1}$ et $\sigma_{3}$ contraintes principales extrêmes,

$\eta \quad$ coefficient de fluidité,

n coefficient de sensibilité à la contrainte. 


\section{3}

\section{Abaque de convergence}

Lè développement semi-explicite du modèle analytique a permis d'établir des abaques adimensionnels de convergence en fonction du temps. La figure 15 englobe les courbes de convergence correspondant à plusieurs valeurs de $n$ pour une structure à température ambiante. Cet abaque sera utilisé ultérieurement pour la détermination des paramètres rhéologiques du sel. Le temps réel est lié au temps adimensionnel par le coefficient $\tau$ défini par la relation suivante:

$$
\tau=\frac{1-v^{2}}{E \eta_{0}} \frac{1}{g_{0}^{n-1}}, \quad g_{0}=2 \mathrm{P}
$$

E: module d'élasticité; $v$ : coefficient de Poisson; P pression lithostatique.

\section{4}

\section{Choix des caractéristiques rhéologiques}

Nous recherchons les caractéristiques rhéologiques de la loi de Norton-Hoff à partir des mesures in situ, de sorte que la réponse du modèle puisse satisfaire les conditions suivantes:

1. donner la même valeur de convergence que celle de la galerie CPPS au bout de quatre années;

2. traduire, comme c'est le cas in situ, une convergence prépondérante dans la première année par rapport aux années suivantes. C'est le paramètre n qui joue le rôle le plus important, du fait qu'on lui attribue une valeur supérieure à 1, exprimant la non linéarité du comportement vis-à-vis de l'état de contraintes;

3. Exprimer également une fluctuation semblable à celle observée in situ qui respecte celle due au changement de la température. Ce sont le module d'élasticité et le coefficient $n$ à mettre en avant pour ce phénomène, le premier affectant le déviateur d'origine thermique (voir l'expression (1) ci-dessus) et le deuxième diminuant le déviateur résiduel d’origine mécanique;

Convergence $\mathrm{E} / \mathrm{g}_{0} \mathrm{u} / \mathrm{r}$

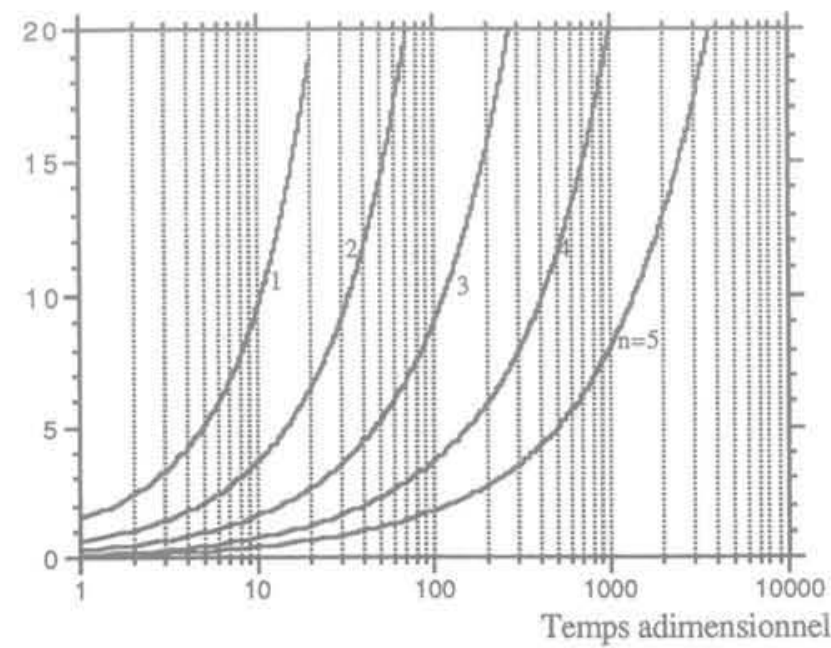

4. respecter les valeurs des paramètres vraisemblables: pression lithostatique $\mathrm{P}$, variation du chargement thermique (valeur mesurée), module d'élasticité et coefficient de dilatation thermique.

Nous procédons dans l'ordre aux opérations suivantes:

1. on obtient la valeur movenne de la convergence $\mathrm{u} / \mathrm{r}$ mesurée au bout de quatre ans, à différentes stations. Elle est de l'ordre de 1,5.10-2 ;

2. on en déduit la convergence adimensionnelle $\mathrm{E} / \mathrm{g}_{0}$ $\mathrm{u} / \mathrm{r}$ où $\mathrm{g}_{0}=2 \mathrm{P}$ est le déviateur de contrainte d'origine mécanique à la paroi ( $\mathrm{P}$ fixé à $10 \mathrm{MPa}$, E est d'environ $15000 \mathrm{MPa}$ ) ce qui revient à trois choix de convergence adimensionnelle comprise entre 11 et 25;

3. en introduisant la valeur de la convergence adimensionnelle dans l'abaque (Fig, 15), on détermine cinq valeurs des temps adimensionnels correspondant à différentes valeurs de $n$;

4. le temps adimensionnel étant lié au temps réel par le coefficient de conversion $\tau$ (le coefficient de Poisson étant fixé à 0,25), on en déduit toutes les cinq valeurs du coefficient de fluidité.

Il en ressort que le choix des parametres permettant de retrouver la convergence mesurée au bout de quatre ans n'est pas unique. En effet, plusieurs combinaisons de paramètres sont possibles. Notre choix porte sur une combinaison des paramètres qui traduit au mieux l'allure concave de la convergence mesurée au début de l'expérience in situ. Ce phénomène va en faveur du choix de grandes valeurs de $n$. Une valeur de $n$ comprise entre 4 et 5 nous semble plausible. Nous retenons une valeur de $n=4,5$ qui, compte tenu d'un module d'Young de $\mathrm{E}=15 \mathrm{GPa}$, revient à un coefficient de fluidité de $\eta=9,610^{-10} / \mathrm{jour}$.

Pour tenir compte de l'influence de la température, un moyen approché consiste à introduire une fonction sinusoidale amortie, issue de l'ajustement des courbes de température mesurées in situ. Cette fonction aura pour période un an: son amplitude sera décroissante dans le massif avec un décalage caractéristique de la diffusion thermique. L'expression exacte de la fonction

\section{Convergence $\mathrm{E} / \mathrm{g}, \mathrm{u} / \mathrm{r}$}

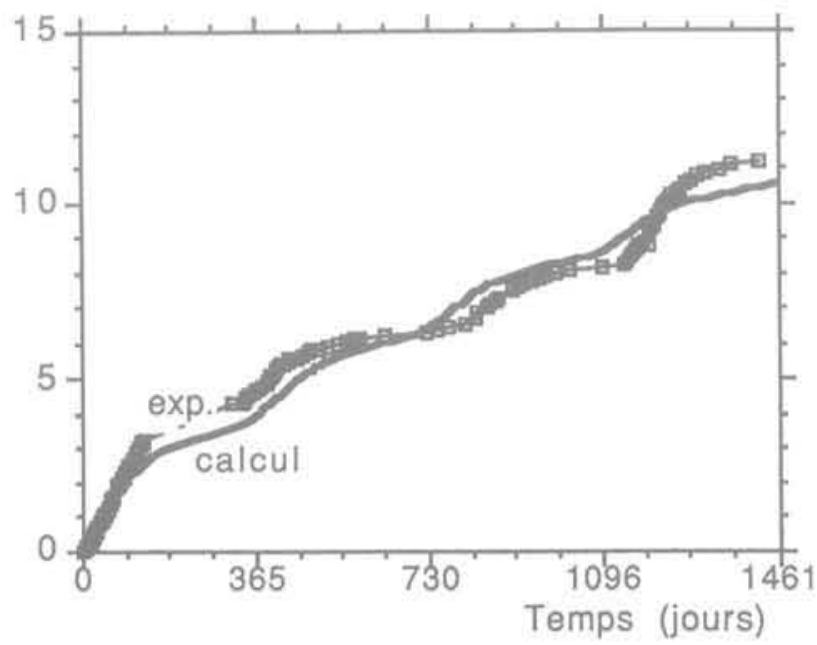

FIG.16 Convergence de la galerie. Closure of CPPS gallery. 
Convergence $\mathrm{E} / \mathrm{g} \mathrm{u} / \mathrm{r}$

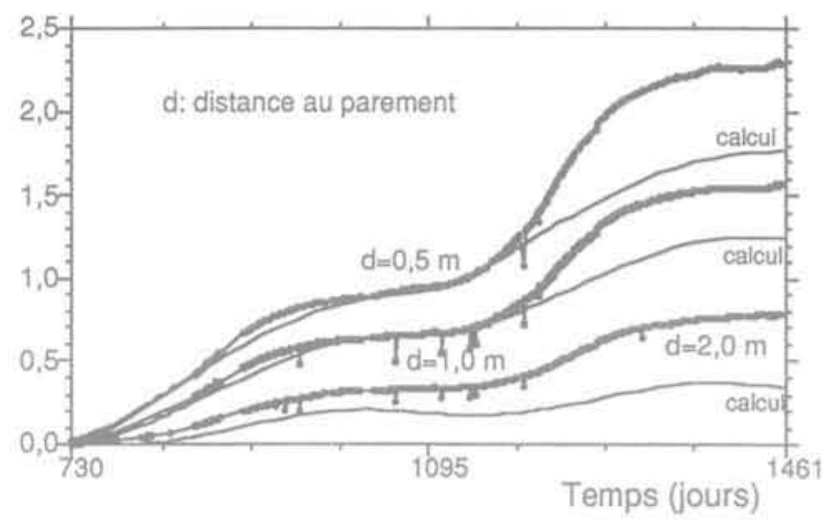

FiG.17 Déplacement dans le massif. Rock mass displacement.

qui pourrait être obtenue analytiquement (expression complexe dépendant de fonctions de Bessel) ne pourrait pas être utilisée facilement dans un calcul thermomécanique. C'est pourquoi nous prenons l'expression empirique suivante ( $\mathrm{r}$ et $\mathrm{t}$ sont le rayon et le temps respectivement):

$$
T(r, t)=f(r) g(t)=\frac{T_{0}}{r^{0,45}}\left(1-\sin \frac{2 \pi}{365} t\right)
$$

La fonction $\mathrm{g}(\mathrm{t})$ est une fonction sinusoìdale dont l'amplitude est mesurée en paroi de la galerie, f(r) est une fonction décroissante en fonction de la distance. La figure 16 illustre l'évolution de la convergence de la galerie ainsi obtenue, qui s'accorde bien avec celle mesurée in situ.

\section{5}

\section{Déplacement et contraintes dans le massif}

La figure 17 montre le déplacement du massif en trois points. L'écart entre les courbes calculées et mesurées, obtenu vers la fin des mesures est dû au chauffage du puits. Quant au point situé à $2 \mathrm{~m}$ de la paroi, nous remarquons un déplacement réversible du point profond. Ceci s'explique par le fait que les points profonds subissent un déplacement périodique dû au comportement thermoélastique prépondérant du massif situé loin de la source de chaleur, en comparaison avec le déplacement viscoplastique. On comprend ainsi l'origine des mouvements réversibles mis en évidence par les mesures d'extension, réversibilité qui n'est pas observée sur les mesures de convergence de la paroi.

La figure 18 montre l'état de contraintes en paroi du massif. Nous remarquons une diminution des contraintes orthoradiale $\sigma_{\theta}$ et axiale $\sigma_{2}$ pour atteindre les états limites d'allure sinusoïdale semblable à celle de la température.

Un autre phénomène important à souligner est l'apparition des contraintes axiales $\sigma$, de traction en paroi de la galerie. Cette traction est faible, compte tenu de l'amplitude de la variation de la température ambiante, mais se rapproche de la résistance en traction du sel gemme (typiquement de $2 \mathrm{MPa}$ ). On explique ainsi le risque d'une fissuration par refroidis-



FiG. 18 Champ de contraintes calculé en paroi de la galerie.

Stress field calculated on the gallery wall.

sement pour des galeries situées à l'entrée d'air, phénomène observé dans certaines mines et carrières souterraines et également rencontré dans certaines galeries aux MDPA.

\section{5}

\section{Conclusion}

Les résultats expérimentaux présentés ici constituent une contribution et une référence expérimentale concernant le comportement des ouvrages souterrains dans les milieux viscoplastiques du type sel gemme.

Les résultats de l'essai in situ réalisé mettent en évidence avant tout combien le comportement mécanique des évaporites est intimement lié à la température, mème si les ouvrages souterrains dans ces milieux sont exposés seulement à une température ambiante dont la fluctuation est habituellement négligée. On ne peut souvent découpler les effets thermiques et mécaniques, les contraintes thermiques résultant de la variation de la température ambiante influant largement sur la vitesse de déplacement du massif.

Par ailleurs, les mesures effectuées depuis le creusement de la galerie expérimentale fournissent une base de réflexion générale quant à l'utilisation des méthodes classiques de dimensionnement des ouvrages souterrains telles que la méthode de convergence-confinement dont l'emploi est délicat dans le cas des évaporites.

Les mesures thermiques, montrant l'homogénéité radiale du champ de température, ont confirmé que le sel gemme est un matériau continu et isotrope, d'un point de vue des propriétés thermiques. Nous avons pu constater que la zone affectée par les variations de la température est assez étendue (supérieure à 5 rayons de la galerie). Les mesures de déplacement ont montré l'importance des effets différés par rapport aux effets à court terme. Toutefois, il a été démontré que les déplacements élastiques ne doivent pas systématiquement être considérés comme négligeables par rapport aux déformations viscoplastiques, surtout dans les zones 
éloignées des parois des structures soumises à une variation de température. Ainsi, supposer que les zones lointaines ne bougent pas n'est pas une hypothèse vraisemblable pour le sel, matériau sans seuil de viscoplasticité. D'où l'intérêt d'ausculter les ouvrages en milieux salifères dès leur creusement.

Nous avons montré qu'en dépit de la géométrie complexe du site de l'essai, les principaux phénomènes observés et les ordres de grandeurs mesurés peuvent être analysés par la loi rhéologique simple de NortonHoff, à partir d'un calcul analytique de cavité circulaire en milieu isotrope et infini. Ce modèle simple a servi comme base méthodologique pour déterminer les caractéristiques rhéologiques du massif à partir des données in situ.
Nous avons vu combien les ouvrages souterrains dans le sel sont sensibles à la température, et avons constaté qu'une faible diminution de température peut engendrer l'arrêt de la convergence d'un ouvrage. Ce phénomène n'est pas dû seulement à une diminution de la viscosité du sel sous température, tel que le prévoit la loi d'Arrhénius. En effet, la température affecte davantage l'état de contrainte, et ce d'autant plus que les caractéristiques thermoélastiques du sel (module d'Young et coefficient de dilatation thermique) sont élevées. Ce phénomène a pour origine l'apparition de contraintes de traction en paroi de cavités, en périodes hivernales. En effet, une faible variation de température modifie considérablement le champ de contraintes régnant dans le massif.

\section{Bibliographie}

Bazargan B. - A remote control system for in situ test. Proc, of the 30th US symp. Rock Mechanics as a quide for Efficient Utilisation of Natural Resources, West Virginia University, Morgantown, 19-22 June 1989.

Ghoreychi M. - Comportement du sel brove sous l'effet d'une source de chaleur dans les sondages réalisés dans une mine de sel. Rapport CCE, éd. EUR 13638, 1991

Ghoreychi M. et al. - Modélisation thermodynamique du champ proche d'un stockage de déchets radioactifs en formations salifères. CR $7^{*}$ congrès international de Mécanique des roches, Aix-la-Chapelle, 1991

GSF, Forsch...- The HAW project: test disposal of highly reactive radiation sources in the Asse salt mine. Rapport CCE. éd. EUR 16688, 1995.
Hardy H.R. - Time-dependent deformation and failure of geological materials. Proc. of the 3rd US symp. on Rock Mechanics, Colorado School of Mines, Golden, 1959, pp. 137-175.

Kazan Y. - Comportement thermo-élastique-viscoplastique des ouvrages souterrains dans le sel gernme. Thèse de doctorat, École des mines de Paris, 1994

Langer M. - The Rheological Behaviour of Rock Salt. Proc. Of the Ist conf. on the Mechanical Behaviour of Salt, Pennsylvania State University, 1981.

Lux K.H., Heusermann S. - Creep tests on rock salt with changing load as a basis for the verification of theoretical material laws. Proc. of the 6th int. Symp. Of Salt, The Salt institute, Alexandria, 1983, vol. 1 pp $417-435$.

Menzel W., Schreiner W. - Zum geomechanischen Verhalten von Stein Salt
Zvershiedenen Lagerstatten der DDR Neue Bergbautechnik, 1977, vol.7. Teil V, pp. 565-571.

Piper D., Lowe D.G.S. - Project COSA. Comparison of geomechanical computer codes for salt. Addendum to COSA, Report CCE EUR 12134 EN, 1989.

Serata S. - Application of continuum mechanics to design of deep potash mines in Canada. Int. Journ. Rock Mechanics of Mining and Sciences, 1964

Vouille G., Tijani S.M., Hugout B. - Le sel gemme en tant que liquide visqueux. Proc. of the 5th int. cong. Rock Mecha nics, Melboune, Australia, 1983, p. D241D246.

Wawersik W.R., Preece D.S. - Creep testing of salt: procedures, problems and suggestions. Proc. of the 1st conf. on the Mechanical Behaviour of Salt, Pennsylvania State University, 1981. 